To be published in Microgravity Science and Technology

Presented at ELGRA Symposium at Santorini, Greece 21-23/09/2005

\title{
Bubble spreading during the boiling crisis: modelling and experimenting in microgravity
}

\author{
V. Nikolayev, ${ }^{1,2, *}$ D. Beysens,,${ }^{1,2}$ Y. Garrabos ${ }^{3}$ C. Lecoutre,${ }^{3}$ and D. Chatain ${ }^{1}$ \\ ${ }^{1}$ ESEME, Service des Basses Températures, DRFMC/DSM/CEA-Grenoble, \\ 17 rue des Martyrs, 38054 Grenoble Cedex 9, France \\ ${ }^{2}$ CEA-ESEME, ESPCI-PMMH, 10, rue Vauquelin, 75231 Paris Cedex 5, France \\ ${ }^{3}$ CNRS-ESEME, Institut de Chimie de la Matière Condensée de Bordeaux, \\ Université de Bordeaux I, Avenue du Dr. Schweitzer, 33608 Pessac Cedex, France
}

(Dated: February 12, 2018)

\begin{abstract}
Boiling is a very efficient way to transfer heat from a heater to the liquid carrier. We discuss the boiling crisis, a transition between two regimes of boiling: nucleate and film boiling. The boiling crisis results in a sharp decrease in the heat transfer rate, which can cause a major accident in industrial heat exchangers. In this communication, we present a physical model of the boiling crisis based on the vapor recoil effect. Under the action of the vapor recoil the gas bubbles begin to spread over the heater thus forming a germ for the vapor film. The vapor recoil force not only causes its spreading, it also creates a strong adhesion to the heater that prevents the bubble departure, thus favoring the further spreading. Near the liquid-gas critical point, the bubble growth is very slow and allows the kinetics of the bubble spreading to be observed. Since the surface tension is very small in this regime, only microgravity conditions can preserve a convex bubble shape. In the experiments both in the Mir space station and in the magnetic levitation facility, we directly observed an increase of the apparent contact angle and spreading of the dry spot under the bubble. Numerical simulations of the thermally controlled bubble growth show this vapor recoil effect too thus confirming our model of the boiling crisis.
\end{abstract}

\section{INTRODUCTION}

During the nucleate boiling, separate gas bubbles form at the heater surface. The liquid phase of the fluid is thus in the direct contact with the heater and the heat transfer rate is very large. This feature explains the high technological importance of boiling in the applications where the high rate cooling of the heater surface is required. Besides the nucleate boiling, another regime of boiling exists: film boiling. During the film boiling, the heater is covered by a quasi-continuous gas film. This film thermally insulates the heater from the liquid and the heat transfer rate is much smaller than in nucleate boiling regime. The transition from nucleate to film boiling thus causes a sharp loss of the heat transfer and the heater can melt down if the heating power is not controlled. This transition is called "Boiling Crisis" (BC), or "CHF phenomenon" since it occurs when the heat flux $q$ from the heater attains a threshold value $q_{C H F}$ called "Critical Heat Flux" (CHF) [1, 2]. In spite of the considerable amount of studies devoted to this important phenomenon, its mechanism is not well understood. This difficulty originates from the violence of the fluid motion during the conventional boiling that on the one hand conceals the mechanisms of bubble growth from detailed observation, and on the other hand hugely complicates direct numerical simulations. Most still unanswered questions concern close vicinity of the heating surface, down to the scale of the foot of a bubble growing at the surface

*email: vadim.nikolayev@cea.fr phone: +33140795826 fax: +33140794523 of the heater. The recent revival of the interest to the microgravity boiling in general and to $\mathrm{BC}$ in particular is related to the development of the engine [3] of the upper stage of Ariane 5 launcher that will need to be restarted under microgravity conditions.

The boiling can be very different since it is dependent on many parameters like system pressure, presence of the externally imposed fluid flow, temperature in the fluid bulk that can be at saturation or below, thermophysical properties of the fluid and the heater etc. In all cases, BC begins in a very thin fluid layer (adjacent to the heater), the thickness of which is small in comparison with the bubble size. The phenomena at this scale "feel" only the averaged values of macroscopic flow variables and thus do not depend on details of the flow regime which should not thus change the BC mechanism. However the contribution of the microscale hydrodynamic motion (that can change in magnitude with the system pressure, see below) to the BC triggering is still unclear.

For the reasons of industrial importance, boiling is mostly studied at low pressures comparing to the critical pressure $p_{c}$ (the pressure of the gas-liquid critical point) of the fluid under study, e.g. for water or freon at atmospheric pressure. $q_{C H F}$ is then large (of the order of several $\mathrm{MW} / \mathrm{m}^{2}$ for water) and the boiling close to it is indeed extremely violent. However, it is well known 1] that $q_{C H F}$ decreases at high pressures where BC can thus be observed at a smaller heat flux. In addition, the thermal diffusivity is smaller in this regime, the bubble growth slows down, and the optical distortions disappear due to the slowness of the fluid motion. From the point of view of the modelling, this slowness permits to simplify the problem by neglecting the hydrodynamic stresses at the bubble interface, the shape of which can then be cal- 
culated in the quasi-static approximation.

\section{FORCE OF VAPOR RECOIL AND BUBBLE SPREADING}

Several groups [4 6] proposed the vapor recoil instability 7] as a reason for BC. Although they did not make clear why and how this instability can induce the spreading of a dry spot, these authors show that the vapor recoil can be an important factor.

Every fluid molecule evaporated from the liquid interface causes a recoil force analogous to that created by the gas emitted by a rocket engine. It pushes the interface towards the liquid side in the normal direction. This force appears because the fluid necessarily expands while transforming from liquid to gas phase. Obviously, the stronger the evaporation rate $\eta$ (mass per time and interface area), the larger the vapor recoil force. The vapor recoil force per unit interface area reads [7] $P_{r}=\eta^{2}\left(\rho_{V}^{-1}-\rho_{L}^{-1}\right)$, where $\rho_{L}\left(\rho_{V}\right)$ signifies liquid (gas) density.

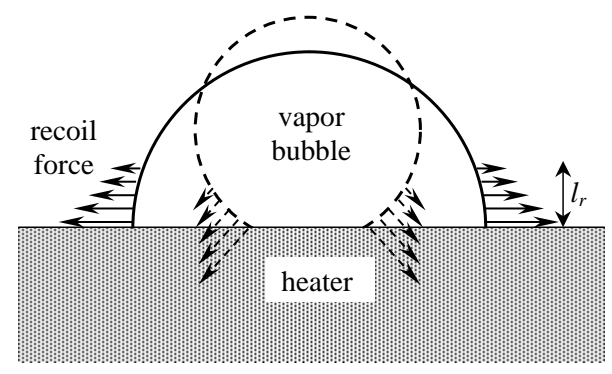

FIG. 1: Sketch illustrating the vapor recoil effect. The amplitude and direction of the vapor recoil force are shown by arrows. The thickness $l_{r}$ of the belt on the bubble surface, in which the vapor recoil force is important, is exaggerated with respect to the bubble size.

Let us now consider a growing vapor bubble attached to the heater surface (Fig. 1). While the temperature of the vapor-liquid interface is constant and equal to the saturation temperature for the given system pressure for the pure fluid (see the discussion in [2, 8, 9] and references therein), a strong temperature gradient forms near the heating surface. The liquid is overheated in a thermal boundary layer, and the heat flux $q_{L}$ at the bubble surface is thus elevated in a "belt" of the bubble surface adjacent to the bubble foot. As a matter of fact, most of the evaporation into the vapor bubble is produced in this belt, whose thickness $l_{r}$ is much smaller than the bubble radius. Since $\eta \approx q_{L} / H$, where $H$ is the latent heat, the vapor recoil near the contact line is much larger than at the other part of the bubble surface. Consequently, the bubble should deform as if the triple contact line were pulled apart from the bubble center as shown in Fig. 1, Therefore, under the action of the vapor recoil the dry spot under the vapor bubble should spread so that the bubble will cover the heater surface.
A numerical simulation [10] of such a process requires solving of coupled thermal conduction (both in solid and liquid) and capillary problems. The capillary problem includes a solution for the bubble shape in the quasistatic approximation in which the local bubble curvature $K$ is defined by the modified Laplace equation $K \sigma=$ $\Delta p+P_{r}$. The bubble shape can be determined from the function $K=K(l)$ (Fig. 2). $\Delta p$ is the constant difference of the pressures in the bubble and in the rest of the fluid and $\sigma$ is the surface tension. $P_{r}$ and then $K$ vary along the interface and attain their maxima near the line of the triple contact liquid-gas-solid. Since curvature is the first derivative of the angle formed by the tangent to the bubble interface and the horizon, the large $K$ means that this angle varies strongly near the contact line. The apparent contact angle $\theta_{a p}$ measured at some distance from the contact line can thus deviate strongly from its actual value $\theta_{e q}$ imposed at the contact line, see Fig. 2

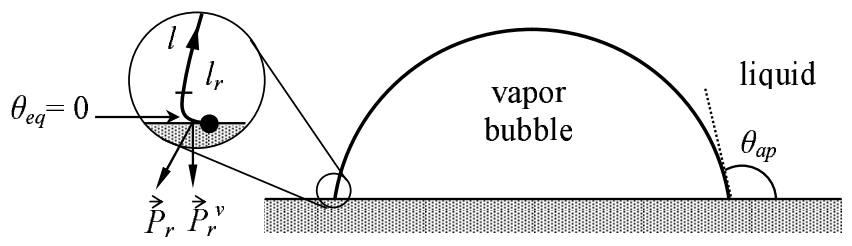

FIG. 2: Apparent $\left(\theta_{a p}\right)$ and actual $\left(\theta_{e q}\right)$ contact angles. The solid circle indicates the contact line, the zero point for the curvilinear coordinate $l$ measured along the bubble contour. The arrows show the directions of the vapor recoil force in the vicinity of the contact line and its vertical component that creates an additional adhesion of the bubble to the heater.

The time of residence of a vapor bubble on the heater is an important parameter since the bubble might simply depart from the heater before having time to spread. The determination of the residence time requires the forces acting on the bubble to be analyzed. Such an analysis 11] shows that the vapor recoil creates an additional adhesion that prevents the bubble departure as soon as the bubble spreading begins, i.e. when the vapor recoil becomes important. This adhesion force can be obtained by integrating the vertical component $P_{r}^{v}$ of $\vec{P}_{r}$ (Fig. 2) over the bubble interface.

The thermal problem is extremely delicate since most of heat transfer occurs at a scale $l_{r}$ much smaller than the bubble size. Extremely fine meshing is thus needed to obtain the correct values of the fluxes near the contact line. Another difficulty appears since the position of one of the solution domain boundaries, that is of the bubble interface, is not known in advance and depends (trough $\left.P_{r}\right)$ on the solution of the thermal problem itself. The solution of the full problem thus needs to be found by time-consuming iterations.

The simulated bubble growth is presented in Fig. 3. It can be seen that the dry spot is initially very small and remains so during the initial growth stage. At about $180 \mathrm{~ms}$ the dry spot begins to grow suddenly, i.e. the bubble spreads. Such a spreading represents the begin- 


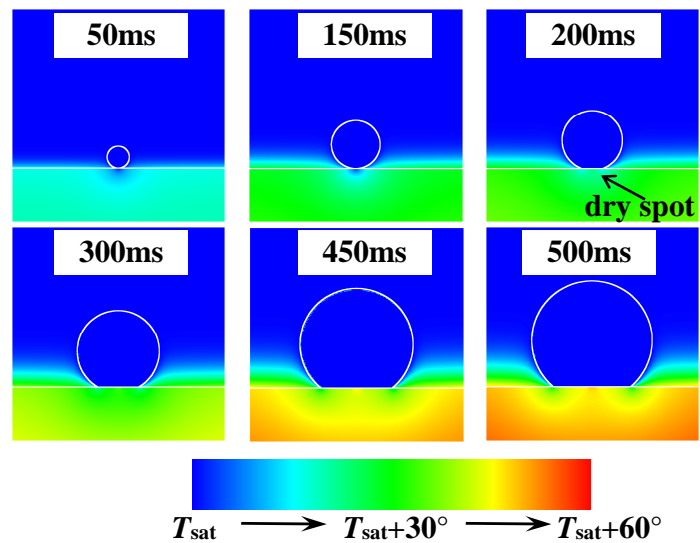

FIG. 3: Vapor bubble spreading under the influence of vapor recoil simulated for water at $10 \mathrm{MPa}$. The color indicates the local temperature with respect to the saturation temperature $T_{\text {sat }}=311^{\circ} \mathrm{C} ; q=0.1 \mathrm{MW} / \mathrm{m}^{2}$.

ning of formation of the gas film characteristic for BC. The apparent contact angle grows sharply while $\theta_{e q}$ is maintained to be zero throughout the simulation. Zero value was chosen because the wetting case is most common. The effect is even stronger for larger contact angles where the dry spot exists from the very beginning. Fig. 3 also shows the formation of a hot spot at the heater surface in the middle of the dry area. This temperature rise illustrates the already discussed blocking of the heat transfer by vapor.

\section{EXPERIMENTS IN REDUCED GRAVITY}

To overcome the experimental difficulties encountered during the observations of $\mathrm{BC}$ at low pressures, we carry out our experiments at very high pressures, in the vicinity of the critical point. We take advantage of the socalled "critical slowing down" observed near the critical point. In fact, due to the smallness of the thermal diffusivity, the growing process of a single vapor bubble could be observed during tens of minutes thus allowing for a very detailed analysis. The $\mathrm{CHF}$ value is also vanishing at the critical point [1] so that BC can be examined at a small heat flux $q$ that does not necessarily induce a strong fluid motion which might hinder the optical observations. However, near-critical bubble growth experiments have an important drawback. Since the surface tension becomes very low near the critical point, gravity completely flattens the liquid interface. Weightlessness conditions are thus necessary to preserve the usual convex bubble shape.

In our already performed experiments, the cells are closed and only pure fluid is present in them so that its total mass and volume remains constant. Unlike the conventional boiling experiments, the gas bubble is not nucleated but exists already before the cell heating begins. The bubble growth is then observed.
Another particularity of the near-critical systems consists in the symmetry of the co-existence curve (temperature dependence of $\rho_{L}$ and $\left.\rho_{V}\right)$ with respect to the critical density $\rho_{c}:\left(\rho_{L}+\rho_{V}\right) / 2=\rho_{c}$. When the average cell density is equal to $\rho_{c}$, the gas volume remains constant and equal to one half of the cell volume throughout the heating. The gas mass however increases (and the liquid mass decreases) during the heating because of the density change. This makes the optical observations even more convenient.

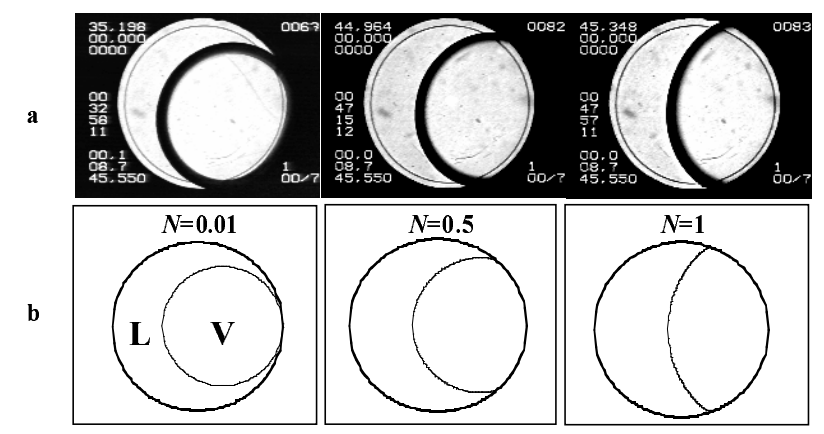

FIG. 4: Snapshots of the vapor bubble (V) growing in near critical liquid (L), both experimental (a) and calculated (b) for the given values of the vapor recoil strength $N$. The zero value of the actual contact angle is imposed while the apparent contact angle attains a large value.

Two series of experiments were carried out up to now. The first [8] used $\mathrm{SF}_{6}$ fluid on board of the Mir space station in the ALICE-2 apparatus designed by the French CNES agency. The choice of $\mathrm{SF}_{6}$ is made for practical reasons: the critical point of this fluid is $T_{c}=45.6^{\circ} \mathrm{C}$, $p_{c}=38$ bar and requires much less severe conditions for the experiment than for example water $\left(374^{\circ} \mathrm{C}, 220 \mathrm{bar}\right)$. Some of the results of this experiment are presented in Fig. 4a. The sequential photos of the growing vapor bubble are taken through the transparent bases of the cylindrical cell, the lateral copper walls of which are being heated. Spreading of the dry spot under the bubble similar to that in Fig. 3 can be seen.

The bubble shapes calculated for different values of the vapor recoil strength $N=\int P_{r}(l) \mathrm{d} l / \sigma$ (where the integration is performed along the bubble contour) are presented for comparison in Fig. 4 $\mathrm{b}$. Only the capillary problem was solved using $\theta_{e q}=0$ as a boundary condition. The $P_{r}(l)$ expression used was that of [12].

However, the cells in ALICE-2 were not suitable to control the heat supply or to measure it. It did not permit to change the bubble position with respect to the cell either. These limitations have been overcome in the experimental $\mathrm{H}_{2}$ setup that makes use of the magnetic levitation facility [13, 14] at CEA-Grenoble. A cylindrical cell of $8 \mathrm{~mm}$ diameter and $5 \mathrm{~mm}$ height is filled with $\mathrm{H}_{2}$ at critical density. The sapphire transparent bases of the cell are good heat conductors in the cryogenic temperature range $\left(T_{c}=33 \mathrm{~K}\right.$ for $\left.\mathrm{H}_{2}\right)$. The lateral cell wall is made of stainless steel which is on the contrary the 
thermal insulator. The fluid is heated by one of the windows while the temperature of the other is maintained by the temperature regulation system which permits us to measure the heat flux removed from the cell. The earth gravity is compensated by magnetic forces within $2 \%$ in the cell volume. The position of the cell with respect to the magnetic field is chosen so that the residual force positions the bubble against the heating window. The growth of the dry spot can thus be directly observed and is illustrated in Fig. 5. Small vapor bubbles grow and depart from the heater under the action of the residual magnetic forces. The bubble spreading begins however for the unique (largest) vapor bubble. One can see that the same mechanism of the dry spot spreading works also relatively far from the critical point. Indeed, the experiments were performed for up to $3 \%$ deviation from $p_{c}$.

\section{CONCLUSIONS}

This paper deals with the vapor recoil model which describes the triggering of the boiling crisis. The results of both simulations and experiments in low gravity confirm this model. However, further studies are necessary. The simulations of the bubble departure under the influence of gravity or/and externally imposed flow should be performed to determine $q_{C H F}$, the heat flux necessary to trigger the boiling crisis. This is a complex problem because of two reasons. First, the heat transfer singularity at the contact line needs to be resolved. Second, the bubble surface position (including the contact line) needs to be determined rigorously, without employing any hypotheses on the interface shape like "liquid microlayer" hypothesis used conventionally [15] to describe the contact line heat transfer.
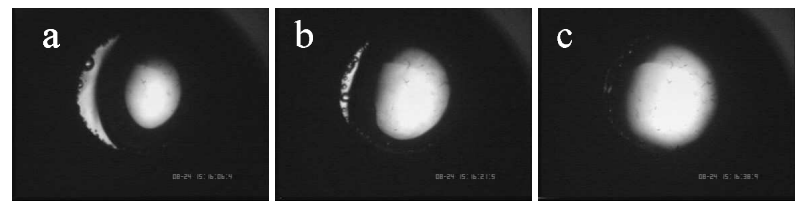

FIG. 5: Gas spreading at 32K (well beyond the critical region) as visualized through the transparent heater in magnetic levitation experiment. (a) Beginning of the dry spot growth. (b) Bubble partially spread. (c) Complete drying of the heater. Nucleated small bubbles (boiling) are visible in (a-b). The volume of the large bubble remains nearly constant throughout the evolution.

The experiments are also needed to be carried on. The drop spreading will be studied quantitatively, both under magnetic levitation and in microgravity on board of the International Space Station in the CNES DECLIC apparatus.

\section{Acknowledgments}

This work was partially supported by CNES. We thank all of the ALICE team and everyone involved into the Mir missions.
[1] Tong, L. S.: Boiling Heat Transfer and Two-Phase Flow (2nd Edn.), Taylor \& Francis, New York (1997).

[2] Straub, J.: Boiling Heat Transfer and Bubble Dynamics in Microgravity, Adv. Heat Transfer, vol. 35, pp. 57-172 (2001).

[3] http://www.snecma-moteurs.com/en/activites/propulsion_sp

[4] Pavlov P. A. E Lipchak A. I., in: Metastable Phase States and Kinetics of Relaxation, Russian Acad. Sci., Ural Division, Sverdlovsk, pp. 119-125 (1992), in Russian.

[5] Avksentyuk, B. P. \& Ovchinnikov, V. V., in: Two-Phase Flow Modelling and Experimentation. Celata, G. P., \& Shah, R. K. (Eds.), Edizioni ETS, pp. 1205-1210 (1995).

[6] Sefiane, K., Benielli, D. \& Steinchen, A.: A new model for pool boiling crisis, recoil instability and contact angle influence. Colloids and Surfaces, vol. 142, pp. 361-373 (1998).

[7] Palmer, H. J.: The hydrodynamic stability of rapidly evaporating liquids at reduced pressure. J. Fluid. Mech., vol. 75 part 3, pp. 487-511 (1976).

[8] Garrabos, Y., Lecoutre-Chabot, C., Hegseth, J., Nikolayev, V. S., Beysens, D., $\&$ Delville J.-P.: Gas spreading on a heated wall wetted by liquid, Phys. Rev. E, vol. 64, 051602 (2001).

[9] Barthes, M., Reynard, C., Santini, R., E Tadrist, L.: Détermination des caractéristiques géométriques de la croissance d'une bulle de vapeur et des transferts de chaleur associés : influence des incondensables sur le déclenchement d'instabilités convectives, Mécanique \& Industries, vol. 6, pp. 257-262 (2005).

[10] Nikolayev, V. S., Beysens, D. A., Lagier, G.-L., 86

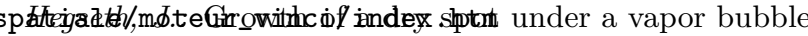
at high heat flux and high pressure. Int. J. Heat Mass Transfer, vol. 44, pp. 3499-3511 (2001).

[11] Nikolayev, V. S., Beysens, D., Garrabos, Y.: Crise d'ébullition : inhibition du détachement de la bulle de vapeur par la force de recul, Mécanique \& Industries, vol. 5, pp. 553-558 (2004).

[12] Nikolayev, V. S. \& Beysens, D. A.: Boiling crisis and non-equilibrium drying transition, Europhysics Letters, vol. 47, pp. 345-351 (1999).

[13] Wunenburger, R., Chatain, D., Garrabos, Y., \& Beysens, D.: Magnetic compensation of gravity forces in (p-) hydrogen near its critical point: Application to weightless conditions, Phys. Rev. E, vol. 62, pp. 469-476 (2000).

[14] Chatain, D., Nikolayev, V. S.: Using magnetic levitation to produce cryogenic targets for inertial fusion energy: experiment and theory, Cryogenics, vol. 42, pp. $253-$ 261 (2002).

[15] Dhir, V.K.: Numerical Simulations of Pool-Boiling Heat Transfer, AIChE Journal, vol. 47, pp. 813-834 (2001). 